\title{
Bionic Control of Cheetah Bounding with a Segmented Spine
}

\author{
Chunlei Wang and Shigang Wang \\ The State Key Laboratory of Mechanical System and Vibration, Shanghai Jiao Tong University, Shanghai 200240, China \\ Correspondence should be addressed to Shigang Wang; wangshigang@situ.edu.cn
}

Received 9 October 2015; Revised 25 January 2016; Accepted 28 January 2016

Academic Editor: Laurence Cheze

Copyright (C) 2016 C. Wang and S. Wang. This is an open access article distributed under the Creative Commons Attribution License, which permits unrestricted use, distribution, and reproduction in any medium, provided the original work is properly cited.

A cheetah model is built to mimic real cheetah and its mechanical and dimensional parameters are derived from the real cheetah. In particular, two joints in spine and four joints in a leg are used to realize the motion of segmented spine and segmented legs which are the key properties of the cheetah bounding. For actuating and stabilizing the bounding gait of cheetah, we present a bioinspired controller based on the state-machine. The controller mainly mimics the function of the cerebellum to plan the locomotion and keep the body balance. The haptic sensor and proprioception system are used to detect the trigger of the phase transition. Besides, the vestibular modulation could perceive the pitching angle of the trunk. At last, the cerebellum acts as the CPU to operate the information from the biological sensors. In addition, the calculated results are transmitted to the low-level controller to actuate and stabilize the cheetah bounding. Moreover, the delay feedback control method is employed to plan the motion of the leg joints to stabilize the pitching motion of trunk with the stability criterion. Finally, the cyclic cheetah bounding with biological properties is realized. Meanwhile, the stability and dynamic properties of the cheetah bounding gait are analyzed elaborately.

\section{Introduction}

As known, cerebellum controls the balance and locomotion of the animals [1]. Meanwhile, the vestibular system and proprioception system also play vital roles for stabilizing the posture and movement of human $[2,3]$. The vestibular system could detect the linear and angular acceleration of body in three dimensions [4]. In addition, with the capability of sensing the gravitational acceleration, the vestibular system could act as a feedback sensor for detecting the body attitude $[5,6]$. The proprioception system is another important feedback sensor. The angle and angular velocity of the joint, the stress of the muscle, and the location of the body segments could be perceived by the proprioception system [7]. Combined with the otoliths' function of detecting the gravity vector, proprioception gives information about body segments configuration and provides clues to be aware of the body general posture without the vision [8].

The effects of the bioinspired control were verified in some robotic systems. He et al. [2] employed the proprioception to capture the output joint angles produced by the central pattern generator (CPG). And then, the achieved data was transferred to the cerebellum for comparing with the parameter values from the cerebral cortex. Finally, the compensation was sent to adjust the CPG network. With the bioinspired control strategy, a human walking gait was realized based on the seven-link biped robot model [9]. Wang et al. [10] built a reflex map between forward velocity and the pitching movement through statistical regression analysis. A velocity controller based on energy control and vestibular reflexes was built by mimicking the way for mammalian to control the body posture. With the bioinspired controller, the robot could adjust the forward velocity by sensing the pitching movement based on the reflex map between the forward velocity and the pitching movement. Fukuoka et al. [11] built a planar model equipped with a CPG consisting of four coupled neural oscillators. In addition, the sensory feedback was introduced in the CPG model to measure the pitching angle of trunk by the vestibular modulation [11, $12]$. Moreover, the proprioceptive sensory feedback was also introduced in the CPG model and used to perceive the phase of the legs [11, 12]. With the vestibular and proprioception modulation, a lateral walking at low speeds, a transverse gallop at a high speed, and a trotting at a medium speed were realized spontaneously and stably. 


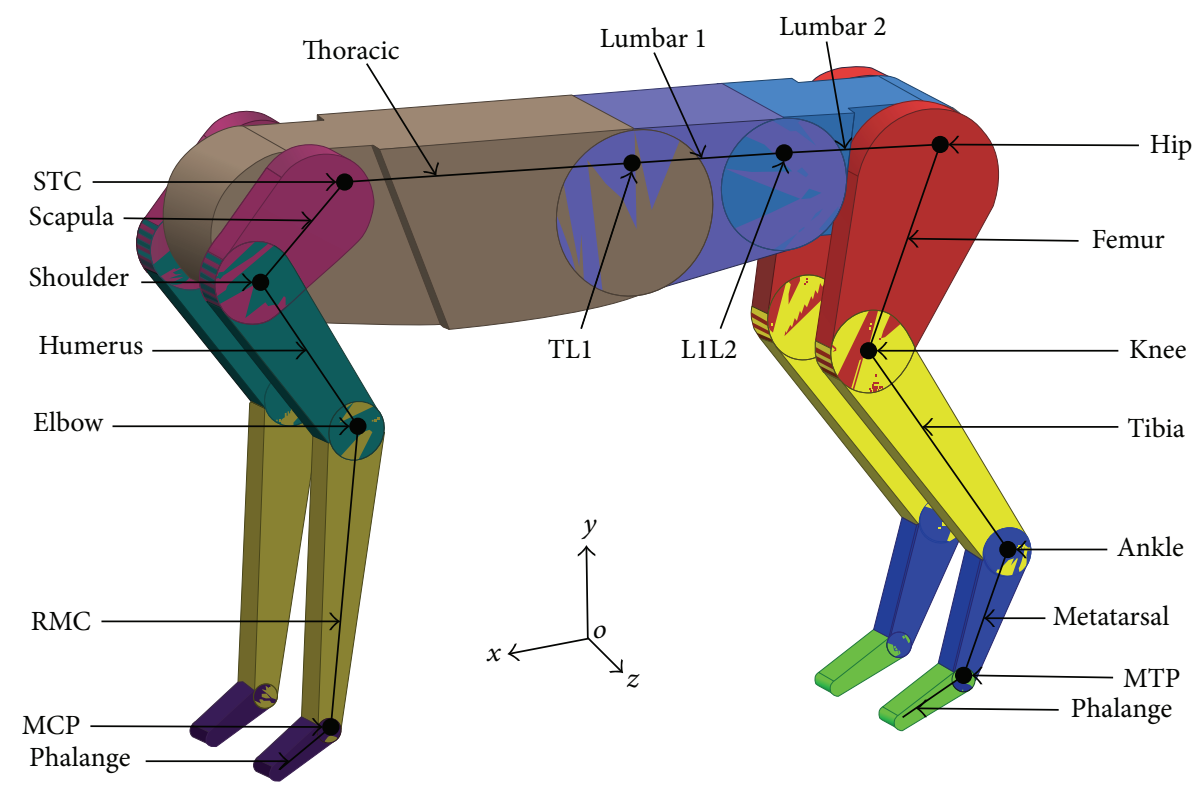

FIGURE 1: Dynamic model of cheetah.

In this study, we present a dynamic model which uses the simplified structures and biological parameters similar to a real cheetah. The articulated spine and legs are the key factors of the model. On the basis of the cheetah model, the bounding gait at a medium speed with biological characteristics is realized by the bioinspired control strategy. We adopt the suggestion in [13]; the bioinspired controller consisted of high-level controller and low-level controller. The high-level controller includes the cerebellum, the vestibular modulation, the proprioception system, and the haptic sensor; its main task is to actuate the cheetah bounding and stabilize the body attitude. With the stability criterion [14], we employ the delay feedback control (DFC) method in the cerebellum to stabilize the planar pitching motion by virtue of the vestibular feedback. In addition, the feedback input determined by the high-level controller should be exerted by the low-level controller which mainly adopts the PD control to actuate the end effectors. Moreover, the proprioception system and haptic sensor of legs could sense the phase of legs. By the bioinspired model and bioinspired control strategy, the bounding gait of cheetah is realized with the motion of spine.

This paper is organized as follows: in Section 2, the dynamic model of cheetah robot is built for mimicking a real cheetah. Then, in Section 3, the bioinspired controller with cerebellum, the vestibular modulation, the proprioception system, the velocity sensor, and the haptic sensor is designed based on the finite state machine. In addition, the stability control algorithm of the cerebellum is described. In the Section 4 , the successive dynamic cheetah bounding is realized, the results will be presented. Additionally, the dynamics of the cheetah bounding will be analyzed, meanwhile, some discussions will be given in Section 5. Finally, in Section 6, we will give some summaries.

\section{Dynamic Model of the Cheetah Robot}

Our object is to build a model that could capture most of the important properties of a real cheetah. The bounding cheetah of Wang et al. [15] is the most detailed cheetah model in simulation to date, however, the coordination motion of spine and legs are the key characteristics of the running cheetah. So on the basis of the bounding cheetah [15], we present a new cheetah model with two actuated spine joints which is shown in Figure 1.

Since the thoracic region is less flexible than the lumbar region [16], the thoracic spine is simplified as a rigid body and its length is equal to the lumbar spine. In addition, the joint between the thoracic and lumbar spine is defined as TL1. Furthermore, the lumbar spine is divided into two equal rigid bodies to simulate the motion in lumbar. The two segments of lumbar are named Lumbar 1 and Lumbar 2 respectively and connected by a joint named L1L2. The joints of forelimb are STC, shoulder, elbow, and MCP. By contrast, the hip, knee, ankle, and MTP are the joints of the hindlimbs from proximal to distal. All the current angles of joints in Figure 1 are zero, and the positive direction of the joint angles is the extension direction. However, the only difference occurs in STC and hip, the positive direction points to the retraction direction. The segments of fore-limbs are scapula, humerus, RMC, and phalange from proximal to distal. By contrast, the segments of the hind-limbs are femur, tibia, metatarsal, and phalange respectively. The length of the segments is defined according to the anatomical measurements of cheetah [17-19]. We set the scale between anatomical and the simulation structure as $1: 1$. The masses are calculated from the dimensions of the segments which are assumed to have constant densities. Combined with the measurement 
TABLE 1: Mechanical parameters of cheetah.

\begin{tabular}{llcc}
\hline \multirow{2}{*}{ Body } & Variables & Mass $(\mathrm{kg})$ & Length $(\mathrm{mm})$ \\
\hline \multirow{3}{*}{ Spine } & Intergirdle distance & - & 760 \\
& Total mass & 50 & - \\
\hline \multirow{4}{*}{ Fore-limb } & Thoracic & 17.21 & 380 \\
& Lumbar 1 & 8.76 & 190 \\
& Lumbar 2 & 7.80 & 190 \\
\hline \multirow{4}{*}{ Hind-limb } & Scapula & 1.23 & 160 \\
& Humerus & 1.28 & 230 \\
& RMC & 1.02 & 360 \\
& Phalange_f & 0.125 & 80 \\
\hline & Femur & 2.345 & 260 \\
& Metatarsal & 1.52 & 310 \\
& Phalange_h & 0.455 & 160 \\
\hline
\end{tabular}

of cheetah mass [20], we round the parameters of the cheetah model as Table 1 .

We construct the 3D cheetah model in ADAMS environment (Figure 1). An impact-Function-based contact model is used to compute the contact forces between the feet and ground [21] (Equation (1))

$$
f=K \cdot \delta^{n}+C \cdot \dot{\delta} .
$$

The stiffness $K$, force exponent $n$, damping coefficient $C$ and penetration depth $\delta$ are $2855 \mathrm{~N} / \mathrm{mm}, 2.57,1.1 \mathrm{~N}-\mathrm{sec} / \mathrm{mm}$ and $0.1 \mathrm{~mm}$ respectively. All coefficients in contact model match the properties of rubber feet on rubber ground. The frictions between feet and ground are defined as Coulomb Friction, and the static and dynamic coefficient of friction are set as 1 and 0.8 respectively.

The coordinate system is defined as Figure 1. The pitching motion is defined as the thoracic spine rotation around axis $z$ according to the convention. The positive direction of axis $x$ is the forward direction of cheetah and the positive direction of axis $y$ is vertical upward. The 3D cheetah model is symmetrical about the sagittal plane $x o y$.

\section{Bioinspired Controller Design}

The finite state machine is adopted to build the bioinspired controller to realize the bionic characteristics of cheetah bounding especially with the spine motion. The phase diagram is shown in Figure 2; although all legs and spine are actuated independently, the motion of them will be coordinated by the phase transition rules in Tables 2 and 3 .

The diagram of the bioinspired controller is shown in Figure 3. The proprioceptive sensory feedback is used to provide the trigger for the phase transitions. We mainly adopt the joint angles of STC and hip, the touch-down of the toes, and the free time to be the triggers. For the hip joint angle could be perceived from the length and the tension of the muscle by the proprioception system [11]. Unlike the use of Taga $[22,23]$ and Fukuoka et al. [12], we only employ the hip angle as the trigger [24] of phase transition rather than

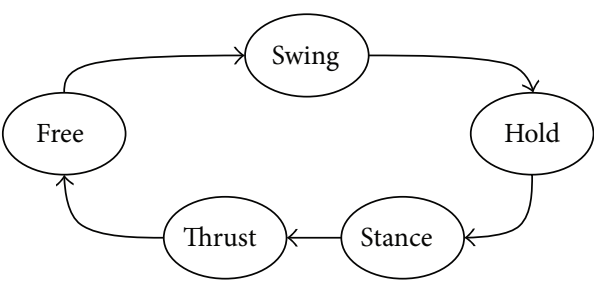

(a)

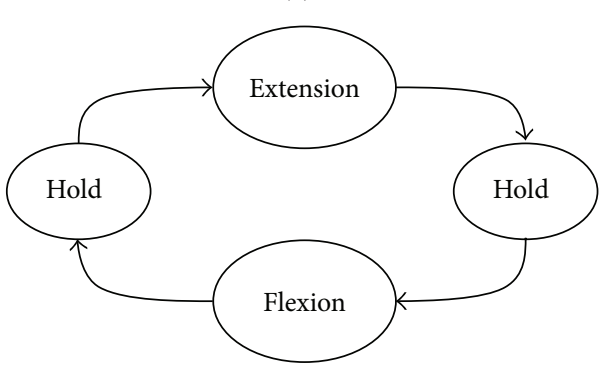

(b)

FIgure 2: Phase diagrams of cheetah bounding controller. (a) Phase diagram for each leg. (b) Phase diagram for spine.

TABLE 2: Phase transition rules of cheetah bounding.

\begin{tabular}{lcc}
\hline Phase transitions & Fore-limb trigger & Hind-limb trigger \\
\hline Thrust to free & $\angle \mathrm{STC} \geq 20^{\circ}$ & $\angle \mathrm{Hip} \geq 20^{\circ}$ \\
Free to swing & $t_{\text {free }} \geq 0.05 \mathrm{~s}$ & $t_{\text {free }} \geq 0.05 \mathrm{~s}$ \\
Swing to hold & $\angle \mathrm{STC} \leq 0^{\circ}$ & $\angle \mathrm{Hip} \leq 5^{\circ}$ \\
Hold to stance & $z_{\text {toe } f} \leq 0$ & $z_{\text {toe } \mathrm{h}} \leq 0$ \\
Stance to thrust & $\angle \mathrm{STC} \geq 15^{\circ}$ & $\angle \mathrm{Hip} \geq 15^{\circ}$ \\
\hline
\end{tabular}

TABle 3: Phase transition rules of spine.

\begin{tabular}{ll}
\hline Phase transition & Trigger \\
\hline Lock to flexion & Forelimb touch-down \\
Flexion to lock & TL1 reaches the minimum limitation \\
Lock to extension & Forelimb lift-off \\
Extension to lock & TL1 reaches the maximum limitation \\
\hline
\end{tabular}

the real time feedback to the CPG model to adjust the motion rhythm. The trigger of transition from hold to stance is the touch-down of the toe which could be perceived by the haptic sensor $\left(z_{\text {toe_f }} \leq 0\right.$ and $\left.z_{\text {toe_h }} \leq 0\right)$. The duration of free phase is hand-tuned according to requirement of the bounding gait.

3.1. Coordination Motion of the Spine and Legs. The motion subcontroller of spine is divided into four phases (Figure 2(b)) which are flexion, hold, extension, and hold [25, 26]. As a result, the motion of spine is concentrated to the ground phase. In this paper, we coordinate the motion of spine and legs by the state transition rules. The phase transition triggers of spine motion are based on the motion limitation of spine and the touch-down and lift-off of the fore-limbs (Table 3). The limitation of the spine motion is detected from the length and the tension of the spine muscle by the proprioception system (Figure 3). The extension stiffness of spine is greater 


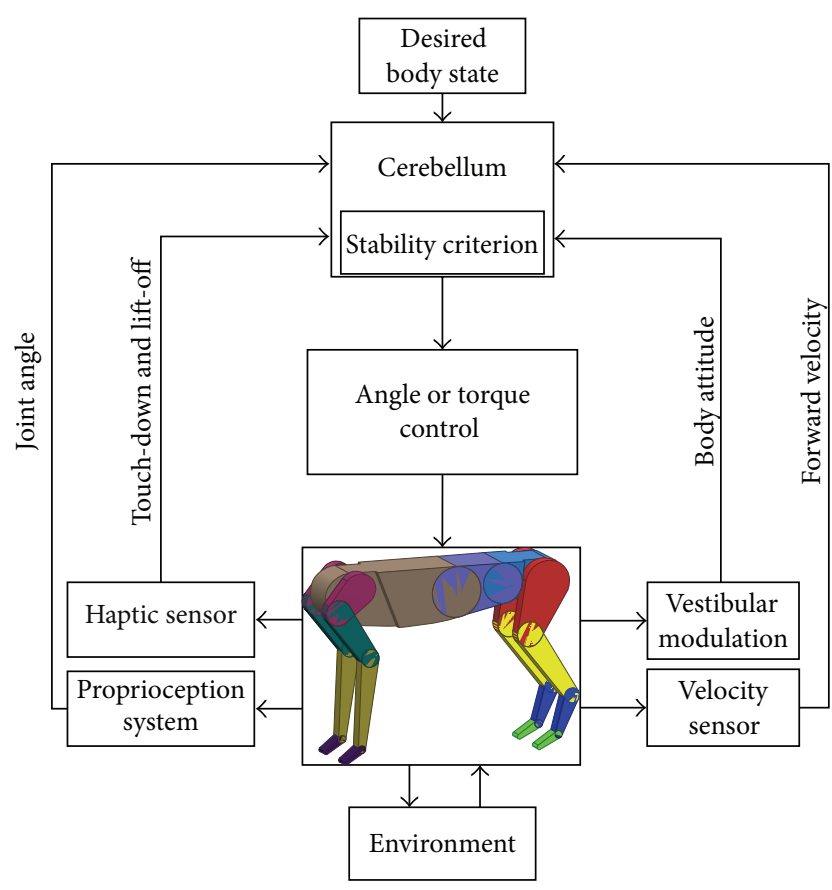

FIGURE 3: The diagram of the bioinspired controller.

than the flexion stiffness [27]; hence, the maximum extension motion of TL1 is set as 0 degrees and the minimum flexion of TL1 is set as -5 degrees artificially. The touch-down and liftoff are detected by the haptic sensor as stated above.

The spine subcontroller switches to flexion phase when the fore-limbs touch down the ground and the spine keeps flexing until the spine joint TL1 reaches the minimum limitation. Next, the lock phase begins, and the spine is locked until the lock phase expires. Next, the extension phase begins when the fore-limbs lift off the ground; the spine keeps extending until the spine joint TL1 achieves the maximum limitation. And, then, the lock phase begins again and the spine is locked until the touch-down of the fore-limbs in the next stride.

The motion equation of spine joints during flexion and extension is simplified as a ramp function:

$$
\theta_{s}=\theta_{s}^{0}+\alpha_{s} \cdot t,
$$

where $s=\mathrm{TL} 1, \mathrm{~L} 1 \mathrm{~L} 2 . \theta_{s}^{0}(\mathrm{rad})$ is the initial angular displacement. $\alpha_{s}(\mathrm{rad} / \mathrm{s})$ is the angular velocity of TL1 and L1L2.

3.2. Flight Phase. During the flight phase, the legs cannot contact with the ground and cannot provide any energy for the bounding. By the proprioception system, the limb joints could retract to the proper angular displacement to prepare for touch-down in the next stride. MCP and MTP joint in fore and hind-limb are compliant joints with a torsional spring and a rotary damper in each joint. As a result, they are passive joints needing no active controller.

3.2.1. Free. In the free phase, to reduce the velocity shock and jerk, the torques of STC and hip are set as zero, so the STC and hip rotate freely. The knee and ankle (shoulder and elbow) are actuated by the planned motion curves to retract from the angular displacement at lift-off to the desired zero-angular displacement:

$$
\begin{aligned}
& \theta_{i}=\theta_{i}^{0}+\alpha_{i} \cdot t, \\
& \alpha_{i}=\frac{\left(0-\theta_{i}^{0}\right)}{t_{\text {free }}},
\end{aligned}
$$

where $i$ is knee, ankle, shoulder, and elbow. $\alpha_{i}(\mathrm{rad} / \mathrm{s})$ is the angular velocity of the joints. $\alpha_{\text {knee }}=\alpha_{\text {ankle }}, \alpha_{\text {shoulder }}=\alpha_{\text {elbow }}$. It is indicated that the motion equation of knee and ankle is the same, as well as the shoulder and elbow. $\theta_{i}^{0}(\mathrm{rad})$ is the initial angle in free phase, that is, the angle when the limbs lift off the ground in thrust phase. The duration time $t_{\text {free }}$ of the free phase is set as $0.05 \mathrm{~s}$, and it is a sufficient time to retract the joints.

3.2.2. Swing. During the swing phase, the knee and ankle (shoulder and elbow) are held at the desired zero-angular displacement. The hip and STC are actuated by the PD control assisted by the sensory feedback of the proprioception system. They swing from the current angle to a desired five and zero degrees (Table 2) in $0.1 \mathrm{~s}$. The swing time is sufficient for limbs to swing and not so less to incur large impacts on the body attitude.

3.2.3. Hold. When the hip or STC reaches the designed transition angles, the controller switches to the hold phase. We simplify the limb preretraction motion before touchdown, so all joints are held on the desired angle of the next touch-down. In the paper, the active controller is adopted to maintain the bounding. As a result, the equal touch-down angle is set from one stride to the next and the forward velocities are planned by the velocity control module during the ground phase.

3.3. Ground Phase. The ground phase is divided into two subphases which are the stance phase and thrust phase according to the functional difference. In the stance phase, the major mission of legs is to provide the torque of STC and hip to keep the forward velocity. On the contrary, the major task of the legs in the thrust subphase is to provide the torque of knee and ankle (shoulder and elbow) to keep the balance of trunk.

3.3.1. Stance. When the fore-limbs touch down the ground, the controller switches to the stance phase of fore-limbs. The major function of the fore-limbs is to touch down smoothly and to provide a transition from one hind-limb's stance phase to another in the next stride.

The velocity sensor detects the current forward velocity; then, the motion control part of the cerebellum could compute the output torque of the STC and hip according to the difference between the current velocity and the target velocity. The torque will be generated by the low-level controller:

$$
T_{j}=\alpha_{j} \cdot\left(v_{\text {target }}-v_{\text {current }}\right)+\beta_{j} \cdot T_{\text {constant }},
$$


where $j=$ Hip, STC, $\alpha_{j}$ is the proportion coefficient which will be hand-tuned, and $T_{\text {constant }}$ is a negative constant torque. $\beta_{j}$ is a coefficient which is used to distinguish the fore and hind-limb:

$$
\beta_{j}= \begin{cases}0 & j=\text { Hip } \\ 1 & j=\text { STC. }\end{cases}
$$

The braking torque exerted on the STC and the thrust torque on the hip could stabilize the pitching motion [28-30]. Therefore, we achieved the pitching stability by introducing a torque bias for STC during the stance phase of fore-limbs. As a result, the torque of STC will become small or even negative when the fore-limbs are in stance phase.

During stance phase, the motion function of knee and ankle (shoulder and elbow) follows (6), and it is also a ramp function of time:

$$
\theta_{l}=\theta_{l}^{0}+\alpha_{l} \cdot t,
$$

where $l$ is knee, ankle, shoulder, and elbow. $\theta_{l}^{0}(\mathrm{rad})$ is the initial angle. $\alpha_{l}(\mathrm{rad} / \mathrm{s})$ is the hand-tuned angular velocity of joints.

3.3.2. Thrust. In the thrust phase, the fore-limbs do nothing except for providing the thrust to generate nose-up motion of the body. The control methods of the STC and hip joints are the same as the stance subphase, and they follow (4) with hand-tuned coefficients in Table 4. The motion of shoulder and elbow will also follow (6) with the hand-tuned coefficients listed in Table 4.

\subsubsection{The Balance Control Algorithm of the Bounding Gait.} During the process of the balance control when the cheetah is dynamic bounding, the high-low level controller is adopted as shown in Figure 3. The cerebellum acts as the CPU of the balance and locomotion control system. The information from vestibular modulation, proprioception system, velocity sensor, and haptic sensor is processed by the cerebellum. In addition, the calculated results are exerted by the low-level controller which mainly adopts the simple PD control to actuate the motion of leg joints. When the cheetah exposes an imbalance posture for a given unstable initial conditions, based on the stability criterion, the controlled force of limbs could be designed to stabilize the bounding gait by adjusting the stiffness of legs [14]. As a result, for the much more complex cheetah model in this paper, the stability criterion [14] could be used as the control algorithm of cerebellum to stabilize the pitching motion of the trunk. The vestibular modulation is used to measure the pitching angle of the thoracic spine at the peak time of the center of mass (COM), that is, the time when the COM is at the maximum vertical displacement. As shown in (7), the difference between zero and the pitching angle at peak time of COM during previous stride is sampled by the vestibular modulation as the control object.

The haptic sensor and proprioception system are used to perceive the phase transitions as stated in Section 3.
TABLE 4: Parameter values of the controller used in simulations.

\begin{tabular}{lccc}
\hline Phase & Parameter & Value & Unit \\
\hline \multirow{2}{*}{ Flexion } & $\alpha_{\text {TL1 }}$ & -1.5 & $\mathrm{rad} / \mathrm{s}$ \\
& $\alpha_{\text {L1L2 }}$ & -2.5 & $\mathrm{rad} / \mathrm{s}$ \\
\hline \multirow{2}{*}{ Extension } & $\alpha_{\text {TL1 }}$ & 1.5 & $\mathrm{rad} / \mathrm{s}$ \\
& $\alpha_{\text {L1L2 }}$ & 2.5 & $\mathrm{rad} / \mathrm{s}$ \\
\hline \multirow{3}{*}{ Stance } & $\alpha_{\text {STC }}$ & 0.01 & - \\
& $\alpha_{\text {hip }}$ & 0.15 & - \\
& $\alpha_{\text {shoulder }} \alpha_{\text {elbow }}$ & 1.2 & $\mathrm{rad} / \mathrm{s}$ \\
& $\alpha_{\text {knee }} \alpha_{\text {ankle }}$ & 1.5 & $\mathrm{rad} / \mathrm{s}$ \\
\hline \multirow{3}{*}{ Thrust } & $\alpha_{\text {STC }}$ & 0.01 & - \\
& $\alpha_{\text {hip }}$ & 0.1 & - \\
& $\alpha_{\text {shoulder }} \alpha_{\text {elbow }}$ & 1.5 & $\mathrm{rad} / \mathrm{s}$ \\
& $\alpha_{\text {knee }} \alpha_{\text {ankle }}$ & 5.3 & $\mathrm{rad} / \mathrm{s}$ \\
\hline & $\delta$ & 1.0 & - \\
& $v_{\text {target }}$ & 2 & $\mathrm{~m} / \mathrm{s}$ \\
& $T_{\text {constant }}$ & -6 & $\mathrm{Nm}$ \\
\hline
\end{tabular}

Moreover, the proprioception system of legs also plays a vital role in the local stability of the limb posture and in maintaining the balance of the trunk. So we adopt the knee and ankle joint as the end effector to exert the control input achieved by the cerebellum. The motion of the knee and ankle is planned by (7).

In the process of actual control, we employ the deviation between the actual pitching angle at the peak time of COM and the zero degrees as the control object. Under the case of the multibody system varies continuously and gradually between two successive strides, a delay feedback control (DFC) method [31] is used to stabilize the pitching motion by controlling the thrust provided by hind-limbs during the thrust phase. The expression of the balance control algorithm of cerebellum is shown in

$$
\begin{aligned}
\theta_{k} & =\theta_{k}^{0}+\left(\alpha_{k}+\Delta \alpha_{k}^{n}\right) \cdot t, \\
\Delta \alpha_{k}^{n} & =\delta \cdot\left(0-\theta_{\text {peak }}^{n-1}\right),
\end{aligned}
$$

where $k$ is knee, ankle, $\alpha_{k}(\mathrm{rad} / \mathrm{s})$ is the basis angular velocity of knee and ankle. $\Delta \alpha_{k}^{n}(\mathrm{rad} / \mathrm{s})$ is the controlled coefficient according to the DFC method, and $n$ indicates the current stride. $\delta$ is the hand-tuned proportional coefficient. $\theta_{\text {peak }}^{n-1}\left({ }^{\circ}\right)$ is the pitching angle at the peak time of COM during the previous stride. $\theta_{k}^{0}(\mathrm{rad})$ is the initial angle which is the last angle of knee and ankle during the stance phase.

The control strategy in this study is the same as [14] which employed a controlled thrust achieved by adjusting the stiffness of legs to compensate the time difference between the zero-time of pitching angle and the peak time of COM. Although the redundant degree of freedom of the leg with multijoints is actuated by planned motion as (7), the output object is the motion and force. The motion and force of point $A$ in Figure 4 are described as follows based on the equations in [32]

$$
F_{N}=\frac{1}{2}\left(\frac{M_{12}-M_{01}}{h_{1}}-\frac{M_{23}+M_{\text {hip }}}{h_{3}}\right),
$$




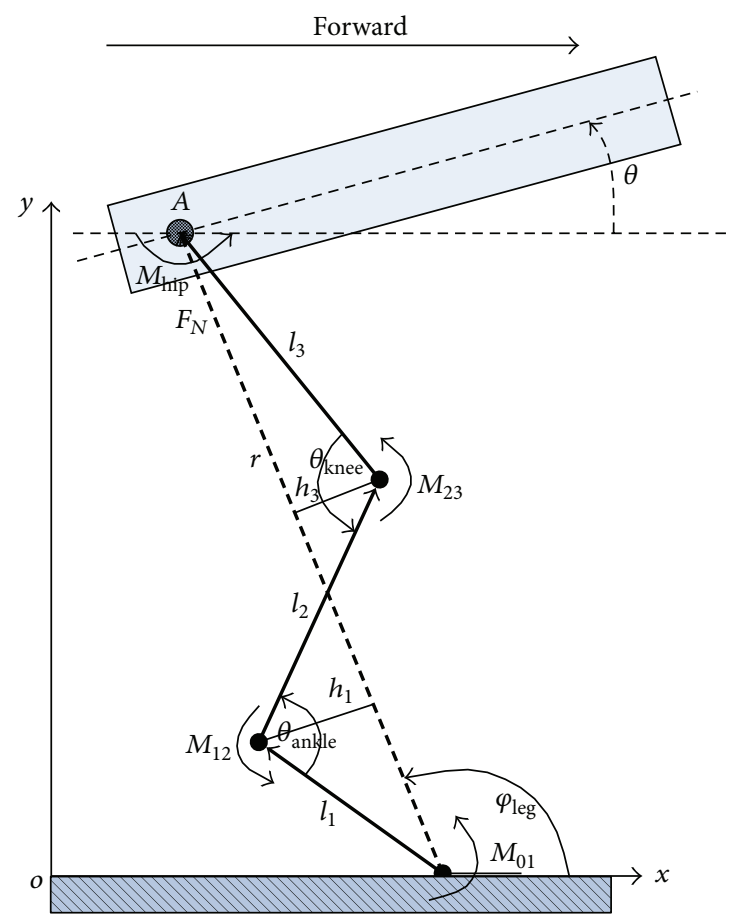

FIGURE 4: Force diagrams of the multi-joint leg.

$$
\begin{aligned}
& h_{1}=\frac{l_{1} l_{2} \sin \theta_{\text {knee }}}{l}, \\
& h_{3}=\frac{l_{2} l_{3} \sin \theta_{\text {knee }}}{l}, \\
& l^{2}=l_{1}^{2}+l_{2}^{2}+l_{3}^{2}-2 l_{1} l_{2} \cos \theta_{\text {knee }}-2 l_{2} l_{3} \cos \theta_{\text {knee }} .
\end{aligned}
$$

The $\theta_{\text {knee }}$ is planned by (7) and $\theta_{\text {knee }}=\theta_{\text {ankle, so, }}$ combining with the controlled $M_{\text {hip }}$ and passive $M_{01}$, the controlled thrust and motion of the hind-limbs are achieved to control the pitching motion.

\section{Results}

We design the bioinspired controller based on the finite state machine by the state-flow in the Matlab/Simulink environment. All the coefficients in Table 4 are hand-tuned in numerous experiments. The multibody dynamics of the cheetah robot are simulated by using the interactive computing between Adams and Simulink.

4.1. The Periodic Bounding Gait of Cheetah. The cheetah robot starts bounding from a stance posture and reaches an ultimate maximum speed of $1.63 \mathrm{~m} / \mathrm{s}$ when the bounding is in the steady state after approximate three seconds. The average forward speed of cheetah is approximate $1.44 \mathrm{~m} / \mathrm{s}$ during the steady state. The cyclic forward velocity is kept by the velocity control module in the cerebellum (Figure 5(a)). The stride frequency is nearly $3 \mathrm{~Hz}$; as a result, the average stride length is $0.48 \mathrm{~m}$. The height of COM achieves a stable periodic state without any control strategy except for the instability and chaos at the starting stage (Figure 5(b)).
The pitching motion also exposes a periodic pattern due to the effect of balance control strategy of cerebellum and the DFC method (Figure 5(c)). Figure 5(d) shows the pitching angle $\theta_{\text {peak }}$ at the peak of COM. The pitching angle deviation from zero at the peak of COM is choppy during the starting stage. At last, $\theta_{\text {peak }}$ is converged to nearly $-0.58^{\circ}$ during the steady state of the bounding gait. According to the passive dynamic analysis and the stability criterion, $\theta_{\text {peak }}$ will converge to zero in the stable bounding gait under the condition of the symmetric body. However, the cheetah model is not a complete symmetry model. And, for instance, the height of the hip at lift-off is higher than the height of the STC at touch-down (Figure 6(b)). As a result, the ascent vertical displacement of COM from lift-off of hind-limbs to the peak is shorter than the descent vertical displacement of COM from the peak to the touch-down of fore-limbs. Therefore, the stable $\theta_{\text {peak }}$ should keep a smaller negative angle to compensate the asymmetric length of the fore and hind-limb (Figure 5(d)). Besides, our cheetah model is not a complete passive model and the energy of the system is nonconservation. For instance, after the hind-limbs lift off the ground, the torque exerted by the hip drives the leg swing forward. As a result, the reactive force on the trunk will increase the nose-down pitching angular velocity. During the same time, the nose-down pitching angle will be larger. So the pitching angle at the peak of COM must be negative to compensate the additionally nose-down motion. In conclusion, the pitching angle at the peak time will be a little negative for our model.

4.2. Sequence of the Bounding Gait. The bounding gait diagrams are shown in Figure 6; meanwhile, the screen captures in the starting of each phase are shown in Figure 7. The angle $\beta$ is the orientation angle of limbs which shows the angle between the line from toe to hip and the vertical line through hip; the retraction direction is the positive direction. Moreover, $z_{\mathrm{STC}}$ and $z_{\text {hip }}$ are the vertical height of STC and hip, respectively. $z_{\text {toe }}$ is the vertical displacement of the toe which could indicate the touch-down and lift-off of the limbs. The total angular excursion of the fore-limbs during ground phase is 12.46 degrees constructed by a protraction angle of -12.26 degrees and a retraction angle of 0.2 degrees. The total angular excursion of the hind-limbs during ground phase is 18.81 degrees which is divided into a protraction angle of -17.75 degrees and a retraction angle of 1.06 degrees. The durations of the fore and hind-limb in ground phase are approximate $0.116 \mathrm{~s}$ and $0.133 \mathrm{~s}$, respectively; the duration of the four limbs' ground phase is $0.016 \mathrm{~s}$. In addition, the stride duration is $0.34 \mathrm{~s}$; consequently, the cheetah contacts with the ground for 69 percent of the total stride.

Figure 7 shows the events in bounding gait of cheetah from the touch-down of fore-limbs which is the starting of a bounding gait to the touch-down of the fore-limbs in the next stride [33]. The cheetah begins flexing the spine when the forelimbs touch down the ground (Figure $7(\mathrm{a})$ ) and the spine keeps flexing until the TL1 joint reaches the minimum limitation. Next, the hind-limbs touch down the ground, so there are four limbs contacting with ground during this time (Figure 7(b)). Then, the spine begins extending when 


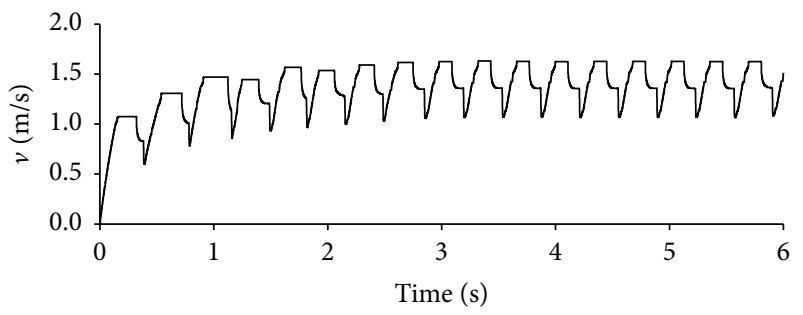

(a)

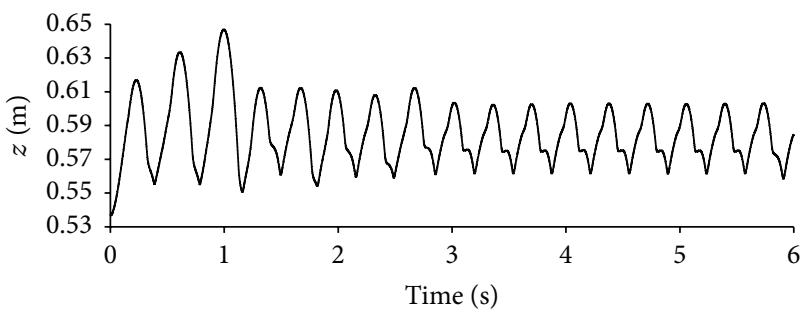

(b)

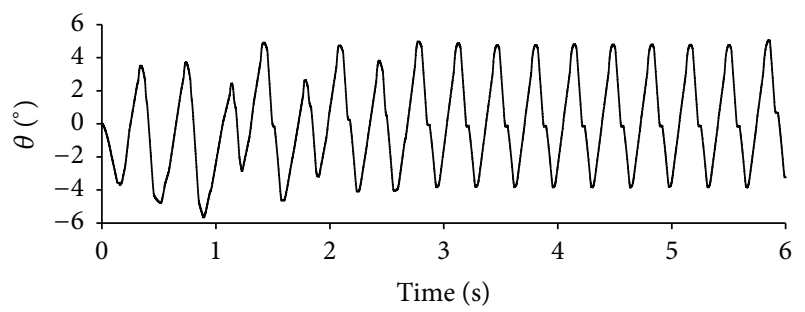

(c)

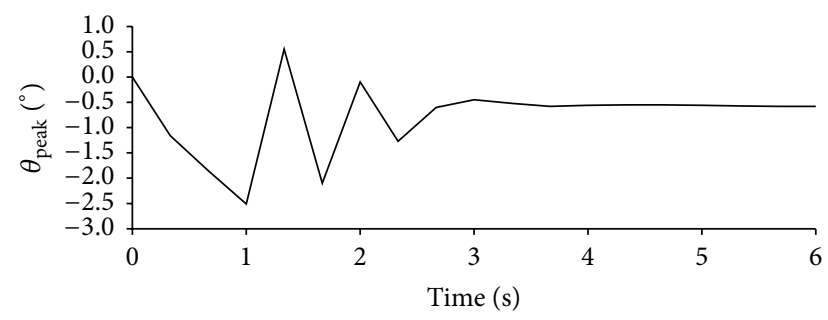

(d)

FIgURE 5: Kinematic variables of the cheetah bounding.

the fore-limbs lift off the ground (Figure 7(c)) and it keeps extending until the TL1 joint reaches the zero degree. And, then, the hind-limbs lift off the ground and switches to the flight phase (Figure $7(\mathrm{~d})$ ). After a transient flight phase (Figure $7(\mathrm{e})$ ), the fore-limbs touch down the ground when the cheetah enters in the next stride (Figure 7(f)).

4.3. Dynamic Analysis of the Bounding Gait. The motions of fore-limb joints, hind-limb joints, and spine joints are shown in Figures 8(d), 8(e), and 8(f). The events (EV) from A to L during a stride are listed in Figure 8 . The forward velocity, height of COM, and pitching angle (PA) will be analyzed over two successive strides (Figures $8(a)-8(c)$ ). One stride is divided into 12 events according to the transition rules. The dynamic variables are affected by the motion of spine and limbs, especially during the ground phase. We employ a small even negative brake torque on the STC to stabilize the pitching motion during the stance phase of the fore-limbs.

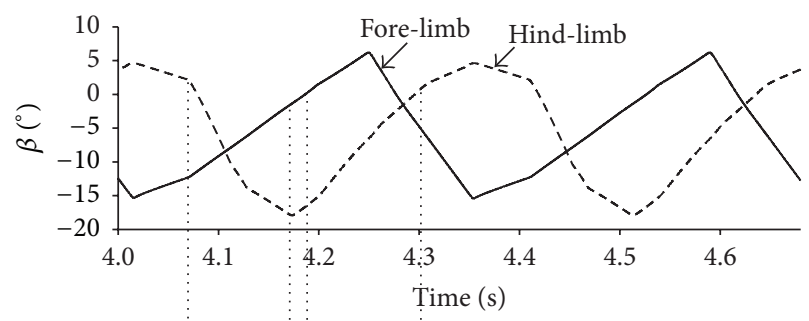

(a)

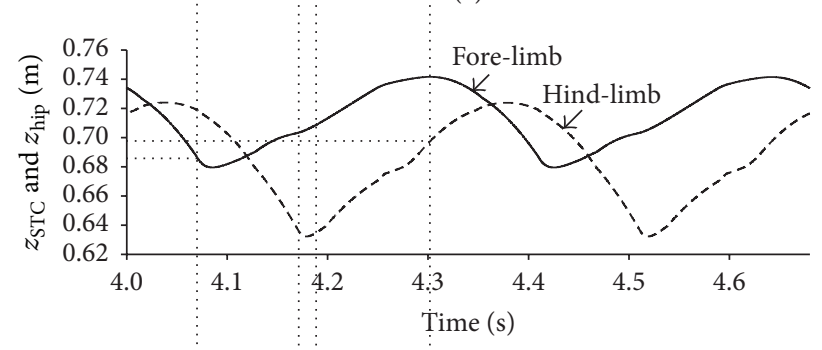

(b)

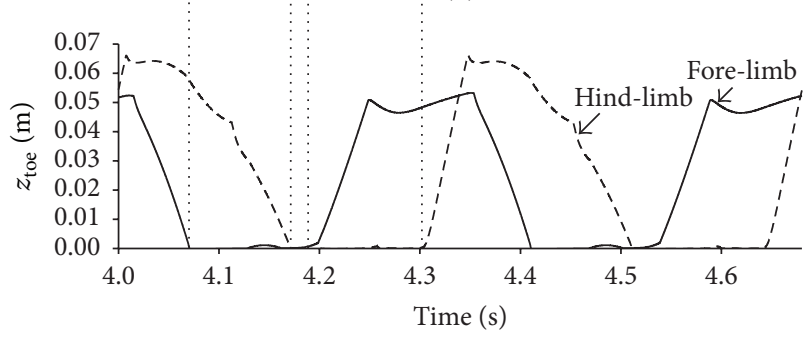

(c)

FIGURE 6: Sequences of the bounding gait.

As a result, the velocity starts to decrease from a constant value when the fore-limbs touch down the ground (EV A). The velocity is even reduced continuously after the hindlimbs touch down the ground $(\mathrm{EV} \mathrm{D})$. The following increased velocity is due to the driving force of hind-limbs. Then, the velocity keeps increasing until the fore-limbs switch from free to swing phase (EV F). The large angular velocity change of STC results in a large angular acceleration, that is, the large torque of STC which makes the hind-foot generate a transient slide. The reason of constant velocity from EV F to $G$ is missing the ground reaction force (GRF). Finally, the velocity of cheetah keeps increasing until the hind-limbs lift off the ground (EV I). Cheetah keeps constant velocity when it is in the flight phase from hind-limbs' lift-off to the next touch-down of fore-limbs.

The maximum vertical movement of the COM during the steady bounding is only $0.041 \mathrm{~m}$ which is a shorter displacement compared with the nominal standing height of $0.537 \mathrm{~m}$; the ratio between them is only $7.6 \%$. Although there is no data about the COM excursion of the bounding cheetah inside or outside laboratory, this ratio is smaller than the galloping horse with the ratio of $9 \%$ [34]. The curve of the vertical displacement of COM is smooth except for the section from EV A to D (Figure 8(b)). The motion of the spine and the brake torque of the STC form a bending spine like an arch. As a result, the vertical excursion of COM forms a local 


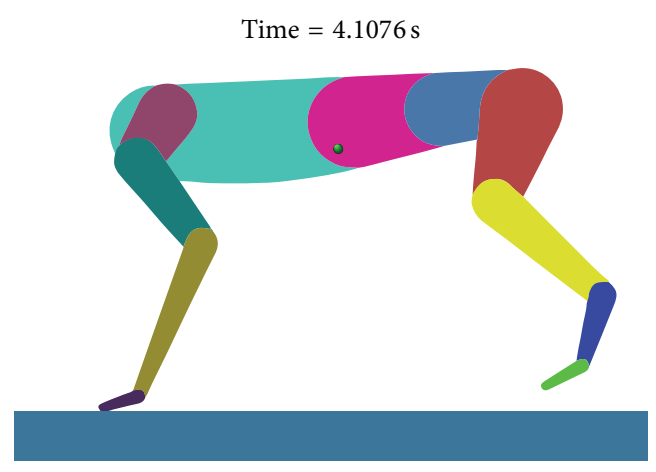

(a)

Time $=4.2239 \mathrm{~s}$

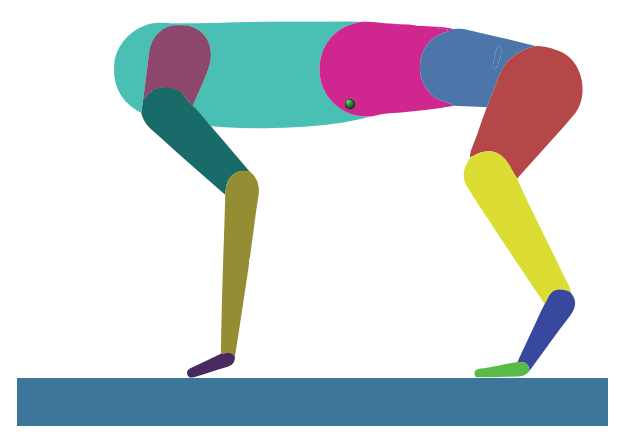

(c)

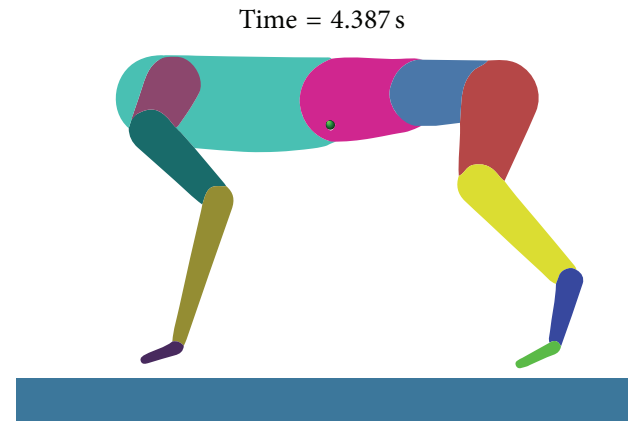

(e)

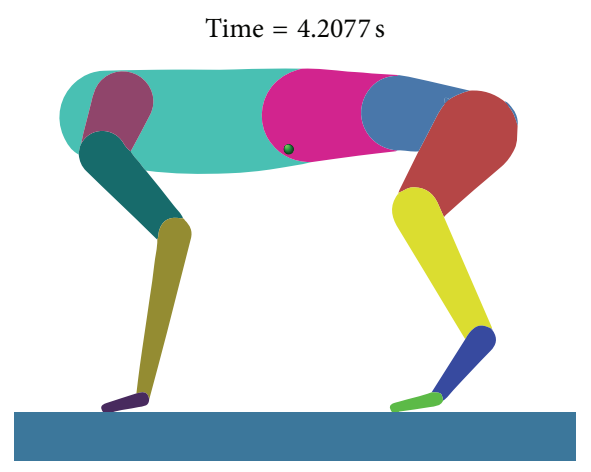

(b)

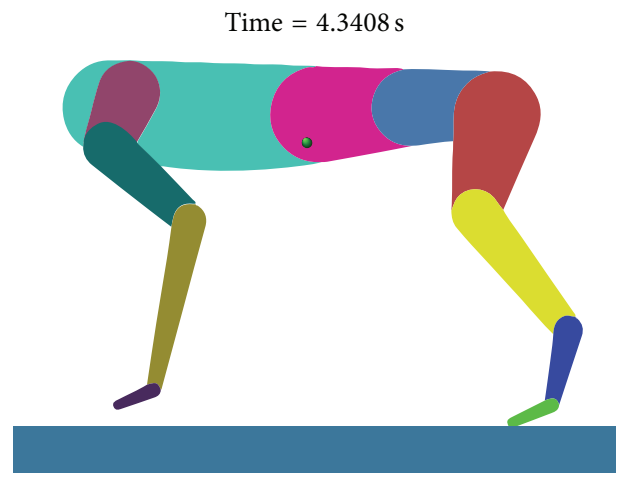

(d)

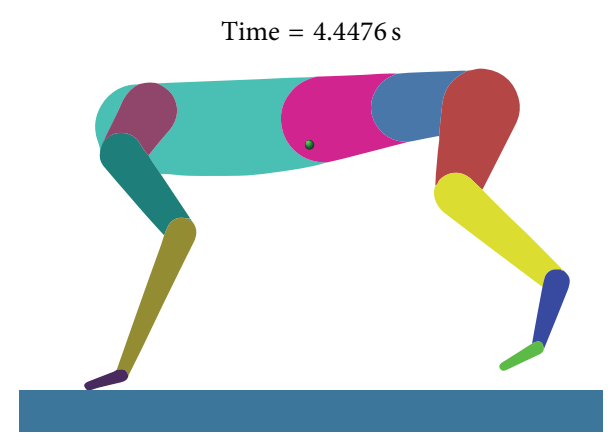

(f)

FIgURE 7: Screen captures of the simulation.

maximum value. The next waves of the curve take place after the spine joints achieve the maximum limitation (EV G). The vertical displacement increases slowly since the displacement incurred by spine motion disappeared (EV G-H). The next regained sharp slope from lift-off of hind-limbs to the peak of COM is derived from the large thrust of hind-limbs during the thrust phase (EV H-I).

When the fore-limbs touch down the ground (EV A), the PA keeps increasing continuously. The phenomenon is attributed to the brake effect of fore-limbs and motion of spine which is the same as we state above for the height wave of COM. After the spine reaches the minimum limitation (EV $\mathrm{C})$, the spine motion is locked; meanwhile, the PA begins decreasing with a nearly constant slope. After the hind-limbs touch down the ground (EV D), PA also presents a local maximum value under the vertical component of hind-limbs'
GRF (EV E). After the fore-limbs lift off the ground (EV E), the trunk of cheetah generates a nose-up motion under the combined effect of spine motion and the torque of hip (EV E$\mathrm{G})$. After the nose-up motion caused by the extension motion of spine is expired (EV G), the trunk begins a nose-down motion driven by the thrust of the hind-limbs. Then, the PA keeps increasing after the hind-limbs lift off the ground (EV I) until the touch-down of the fore-limbs in the next stride (EV L).

\section{Discussions}

5.1. Validity of the Controller under Different Forward Velocities. As shown in Figure 9, the cheetah could run with three different speeds under the bounding gait. The three corresponding parameter sets are listed in Table 5. The bounding 

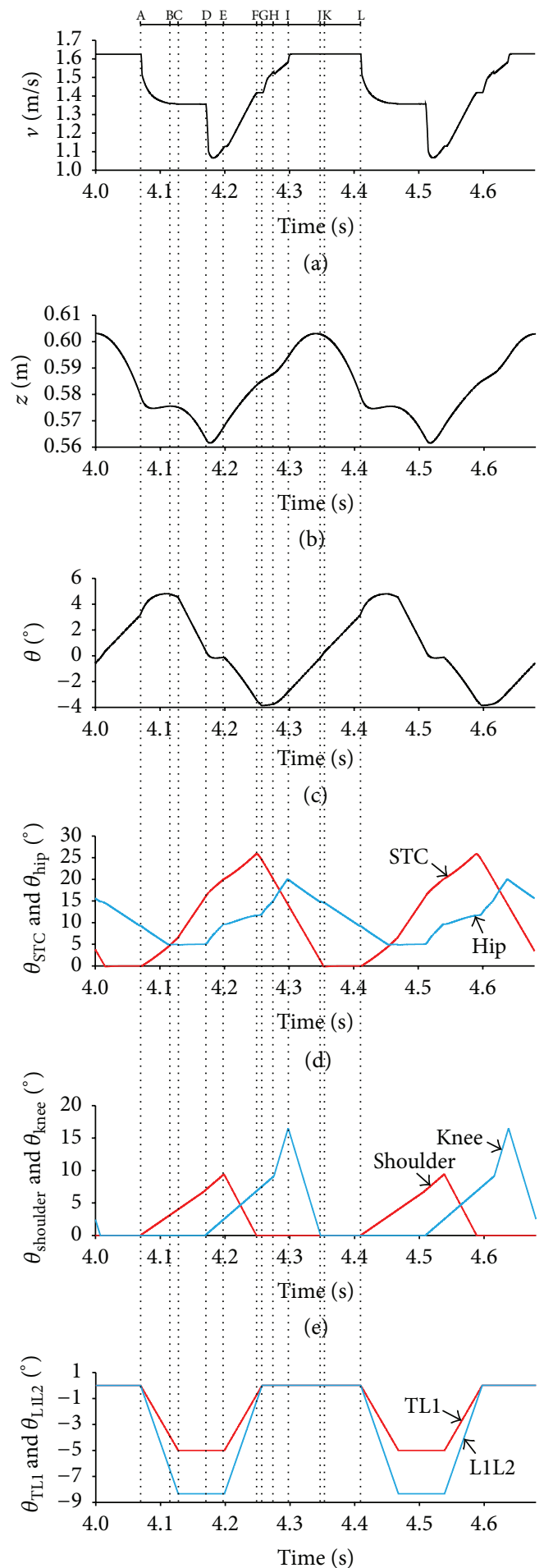

(f)

FIGURE 8: Kinematic variables in the bounding gait over two strides. EV A: Touch-down of fore-limbs. EV B: Transition of hind-limbs from Swing to Hold. EV C: Minimum limitation of the spine joints. EV D: Touch-down of hind-limbs \& Transition of fore-limbs from Stance to Thrust. EV E: Lift-off of fore-limbs. EV F: Transition of fore-limbs from Free to Swing. EV G: Maximum limitation of the spine joints. EV H: Transition of hind-limbs from Stance to Thrust. EV I: Lift-off of hind-limbs. EV J: Transition of hind-limbs from Free to Swing. EV K: Transition of fore-limbs from Swing to Hold. EV L: Touch-down of the fore-limbs in the next stride.

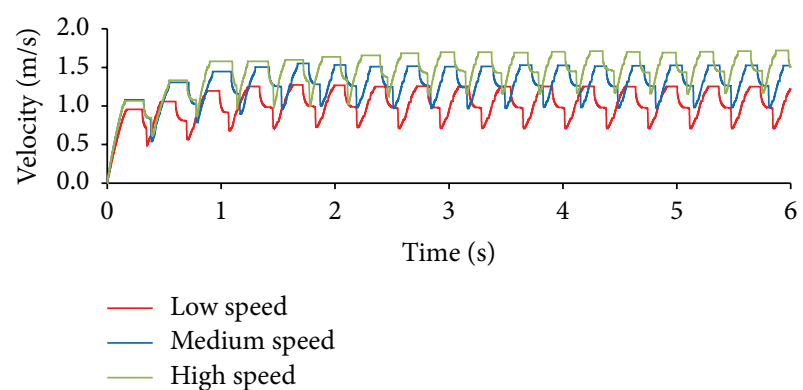

(a)

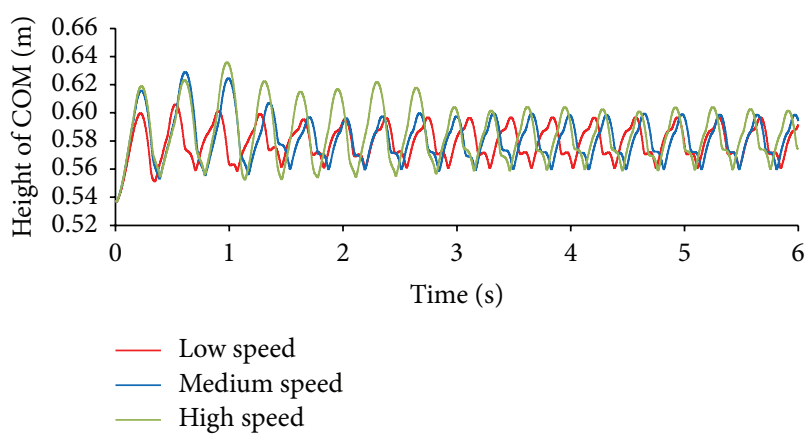

(b)

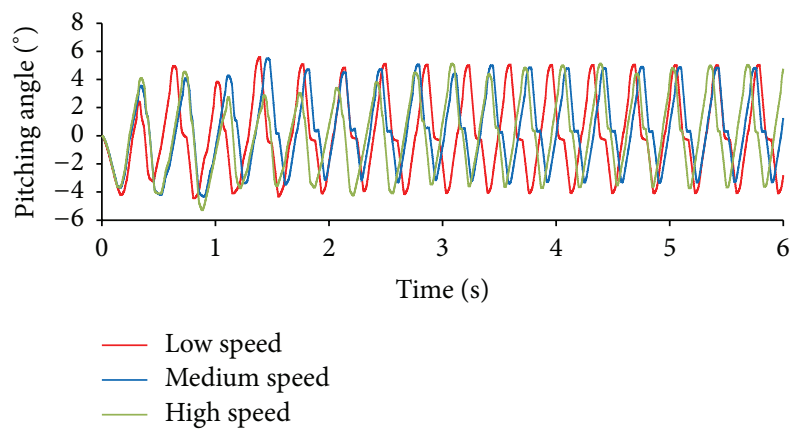

(c)

FIgURE 9: Kinematic variables under different parameters.

gait exposes cyclic feature after 3 seconds. From Figure 9(a), the variation trends of the three speeds are nearly the same; however, they have different specific values and different frequencies. The low, medium, and high speeds are approximate average $1.05,1.33$, and $1.53 \mathrm{~m} / \mathrm{s}$, respectively, during the steady cyclic state. In addition, there are 16, 17 , and 18 complete strides under the low, medium, and high speed, respectively. The proportion coefficients $\alpha_{j}$ between the torque and the velocity difference (Equation (4)) should be increased for increasing the forward velocity during stance phase (Table 5). In addition, $T_{j}$ also shows an increasing tendency and forms an increasing resultant torque of the STC combined with the increasing $\alpha_{j}$. Meanwhile, the leg motion should be planned again to compensate the unstable pitching motion along with the increased forward velocity. The extending angular velocity of shoulder and elbow exposes a decreasing tendency for increasing the forward speed. On the contrary, the extending angular velocity of the knee and ankle shows an increasing tendency for increasing the forward speed. 
TABLE 5: Parameter values of different velocities.

\begin{tabular}{|c|c|c|c|c|c|}
\hline \multirow{2}{*}{ Phase } & \multirow{2}{*}{ Parameter } & \multicolumn{3}{|c|}{ Value } & \multirow{2}{*}{ Unit } \\
\hline & & Low speed & Medium speed & High speed & \\
\hline \multirow{2}{*}{ Flexion } & $\alpha_{\mathrm{TL} 1}$ & -1.5 & -1.5 & -1.5 & $\mathrm{rad} / \mathrm{s}$ \\
\hline & $\alpha_{\mathrm{L} 1 \mathrm{~L} 2}$ & -2.5 & -2.5 & -2.5 & $\mathrm{rad} / \mathrm{s}$ \\
\hline \multirow{2}{*}{ Extension } & $\alpha_{\mathrm{TL} 1}$ & 1.5 & 1.5 & 1.5 & $\mathrm{rad} / \mathrm{s}$ \\
\hline & $\alpha_{\text {L1L2 }}$ & 2.5 & 2.5 & 2.5 & $\mathrm{rad} / \mathrm{s}$ \\
\hline \multirow{4}{*}{ Stance } & $\alpha_{\mathrm{STC}}$ & 0.01 & 0.01 & 0.012 & - \\
\hline & $\alpha_{\text {hip }}$ & 0.08 & 0.12 & 0.186 & - \\
\hline & $\alpha_{\text {shoulder }} \alpha_{\text {elbow }}$ & 1.5 & 1.2 & 1.2 & $\mathrm{rad} / \mathrm{s}$ \\
\hline & $\alpha_{\text {knee }} \alpha_{\text {ankle }}$ & 1.2 & 1.5 & 1.5 & $\mathrm{rad} / \mathrm{s}$ \\
\hline \multirow{7}{*}{ Thrust } & $\alpha_{\mathrm{STC}}$ & 0.01 & 0.01 & 0.01 & - \\
\hline & $\alpha_{\text {hip }}$ & 0.06 & 0.1 & 0.1 & - \\
\hline & $\alpha_{\text {shoulder }} \alpha_{\text {elbow }}$ & 1.8 & 1.6 & 1.5 & $\mathrm{rad} / \mathrm{s}$ \\
\hline & $\alpha_{\text {knee }} \alpha_{\text {ankle }}$ & 4.5 & 5.1 & 5.5 & $\mathrm{rad} / \mathrm{s}$ \\
\hline & $\delta$ & 1 & 1 & 1 & - \\
\hline & $v_{\text {target }}$ & 2 & 2 & 2 & $\mathrm{~m} / \mathrm{s}$ \\
\hline & $T_{\text {constant }}$ & -12 & -7.5 & -4.5 & $\mathrm{Nm}$ \\
\hline
\end{tabular}

The three curves of the COM displacement under different velocities also show the same fluctuation pattern during the steady state (Figure 9(b)). However, the higher forward velocity is, the more time is needed to achieve the steady cyclic gait. In addition, the bounding gait will show the larger oscillations during the chaos state of the starting stage. At last, although there is potential better $\delta$ to choose, the pitching motions of the trunk are stabilized with the same proportion factor $\delta$ (Table 5). The achieved curves of the pitching angles are shown in Figure 9(c). Although there are other parameter sets to make the bounding gait stable, we choose the three of them in Table 5 as the instances. In conclusion, the validity of the bionic controller is verified against different forward velocities and different parameter sets.

5.2. Practicability of the Stability Criterion. The pitching angle is sampled at the peak of COM after the ground phase. Moreover, the lift-off of hind-limbs is the final time of the ground phase. That is, no control action of legs could be used to stabilize the body posture during the flight phase. Inspired by the mammalian vestibular reflex for body posture control, the DFC is a suitable method to stabilize the body posture [10]. Under the case of the system varying continuously and gradually between two successive strides, the previous state variables could be sampled as the control input to calculate the output thrust of hind-limbs. By combining the mammalian nervous system and the stability criterion, the controller could adjust the imbalance posture in real time.

We adopt a spine with two joints to mimic the spine motion of cheetah (Figure 1). The maximum angle of two spine joints is set as zero degrees as the constraint condition. In addition, spine joints reach the maximum limitation before the hind-limbs lift off the ground and keep locked during the flight phase; that is, the spine is rigid during flight (Figures $8(\mathrm{~d}), 8(\mathrm{e})$, and $8(\mathrm{f})$ ). As a result, although the stability criterion derived from the passive beam and spring legs model with a rigid trunk is simple [14], it is applicable for the complex $3 \mathrm{D}$ cheetah model with a segment spine in this paper.

As stated in [14], the stability criterion is as follows: the closer are the two moments which are the zero-time of pitching angle and the peak time of the COM, the better is the stability of the trunk during bounding. Besides, within a certain range, the degree of instability varies monotonously with the deviation degree between the zero-time of pitching angle and the peak time of COM. Therefore, the deviation of pitching angle from zero at the peak time of COM could be defined as the control input. Finally, the achieved stable bounding gait demonstrates the validity of the linear transfer function between the input deviation and the output control action (Equation (7)).

\section{Conclusions}

A simulation model is built with multijoint spine and limbs which can mimic the real cheetah. In addition, the mechanical and dimension parameters of the model are the same as the real cheetah. Besides, for actuating and stabilizing the bounding gait of cheetah, the bioinspired controller is presented based on the state-machine. The controller adopts high-low level control, and the cerebellum is the CPU of the balance and motion planning system. The information of pitching angle at the peak of the flight phase is achieved by the vestibular modulation. The haptic sensor and proprioception system are used to perceive the phase transitions. The calculated results from cerebellum are transmitted to end effector to stabilize the pitching motion and maintain the forward velocity of cheetah. The forward velocity is kept by exerting the controlled torque on the hip and STC. Besides, the pitching motion is stabilized by a DFC method with the stability criterion. At last, the cyclic steady cheetah bounding gait with the actuated spine joints is achieved. 
In addition, the kinematic and dynamic characteristics of the bounding gait are analyzed elaborately. The validity of the bioinspired controller and the stability criterion derived from the simplified passive dynamic model are verified for the more complex cheetah model.

\section{Conflict of Interests}

The authors declare that there is no conflict of interests regarding the publication of this paper.

\section{Acknowledgment}

This project is supported by National Natural Science Foundation of China (Grant no. 51375303).

\section{References}

[1] S. M. Morton and A. J. Bastian, "Cerebellar control of balance and locomotion," The Neuroscientist, vol. 10, no. 3, pp. 247-259, 2004.

[2] B. He, Q. Lu, and Z. Wang, "Coupling effect analysis between the central nervous system and the CPG network with proprioception," Robotica, vol. 33, no. 6, pp. 1281-1294, 2015.

[3] T. G. Deliagina, P. V. Zelenin, I. N. Beloozerova, and G. N. Orlovsky, "Nervous mechanisms controlling body posture," Physiology and Behavior, vol. 92, no. 1-2, pp. 148-154, 2007.

[4] C. Lopez and O. Blanke, "The thalamocortical vestibular system in animals and humans," Brain Research Reviews, vol. 67, no. 1-2, pp. 119-146, 2011

[5] J. F. Iles, R. Baderin, R. Tanner, and A. Simon, "Human standing and walking: comparison of the effects of stimulation of the vestibular system," Experimental Brain Research, vol. 178, no. 2, pp. 151-166, 2007.

[6] A. Berthoz, The Brain's Sense of Movement, Harvard University Press, Cambridge, Mass, USA, 2002.

[7] R. J. van Beers, A. C. Sittig, and J. J. Denier van der Gon, "The precision of proprioceptive position sense," Experimental Brain Research, vol. 122, no. 4, pp. 367-377, 1998.

[8] J. R. Lackner and P. DiZio, "Vestibular, proprioceptive, and haptic contributions to spatial orientation," Annual Review of Psychology, vol. 56, no. 1, pp. 115-147, 2005.

[9] G. Taga, "A model of the neuro-musculo-skeletal system for human locomotion," Biological Cybernetics, vol. 73, no. 2, pp. 97-111, 1995.

[10] X. Wang, M. Li, W. Guo, P. Wang, and L. Sun, "Velocity control of a bounding quadruped via energy control and vestibular reflexes," Journal of Bionic Engineering, vol. 11, no. 4, pp. 556571, 2014.

[11] Y. Fukuoka, Y. Habu, and T. Fukui, "Analysis of the gait generation principle by a simulated quadruped model with a CPG incorporating vestibular modulation," Biological Cybernetics, vol. 107, no. 6, pp. 695-710, 2013.

[12] Y. Fukuoka, H. Kimura, and A. H. Cohen, "Adaptive dynamic walking of a quadruped robot on irregular terrain based on biological concepts," The International Journal of Robotics Research, vol. 22, no. 3-4, pp. 187-202, 2003.

[13] A. J. Ijspeert, "Central pattern generators for locomotion control in animals and robots: a review," Neural Networks, vol. 21, no. 4, pp. 642-653, 2008.
[14] C. Wang, T. Zhang, X. Wei, Y. Long, and S. Wang, "Dynamic imbalance analysis and stability control of galloping gait for a passive quadruped robot," Applied Bionics and Biomechanics, vol. 2015, Article ID 479615, 17 pages, 2015.

[15] X. Wang, M. Li, P. Wang, W. Guo, and L. Sun, "Bio-inspired controller for a robot cheetah with a neural mechanism controlling leg muscles," Journal of Bionic Engineering, vol. 9, no. 3, pp. 282293, 2012.

[16] H. Rockwell, F. G. Evans, and H. C. Pheasant, “The comparative morphology of the vertebrate spinal column. Its form as related to function," Journal of Morphology, vol. 63, no. 1, pp. 87-117, 1938.

[17] P. E. Hudson, S. A. Corr, R. C. Payne-Davis, S. N. Clancy, E. Lane, and A. M. Wilson, "Functional anatomy of the cheetah (Acinonyx jubatus) forelimb," Journal of Anatomy, vol. 218, no. 4, pp. 375-385, 2011.

[18] P. E. Hudson, S. A. Corr, R. C. Payne-Davis, S. N. Clancy, E. Lane, and A. M. Wilson, "Functional anatomy of the cheetah (Acinonyx jubatus) hindlimb," Journal of Anatomy, vol. 218, no. 4, pp. 363-374, 2011.

[19] L. M. Day and B. C. Jayne, "Interspecific scaling of the morphology and posture of the limbs during the locomotion of cats (Felidae)," Journal of Experimental Biology, vol. 210, no. 4, pp. 642-654, 2007.

[20] P. E. Hudson, S. A. Corr, and A. M. Wilson, "High speed galloping in the cheetah (Acinonyx jubatus) and the racing greyhound (Canis familiaris): spatio-temporal and kinetic characteristics," The Journal of Experimental Biology, vol. 215, no. 14, pp. 24252434, 2012.

[21] H. M. Lankarani and P. E. Nikravesh, "Continuous contact force models for impact analysis in multibody systems," Nonlinear Dynamics, vol. 5, no. 2, pp. 193-207, 1994.

[22] G. Taga, "A model of the neuro-musculo-skeletal system for human locomotion. I. Emergence of basic gait," Biological Cybernetics, vol. 73, no. 2, pp. 97-111, 1995.

[23] G. Taga, "A model of the neuro-musculo-skeletal system for human locomotion II. Real-time adaptability under various constraints," Biological Cybernetics, vol. 73, no. 2, pp. 113-121, 1995.

[24] J. H. J. Allum, B. R. Bloem, M. G. Carpenter, M. Hulliger, and M. Hadders-Algra, "Proprioceptive control of posture: a review of new concepts," Gait \& Posture, vol. 8, no. 3, pp. 214-242, 1998.

[25] Q. Deng, S. Wang, W. Xu, J. Mo, and Q. Liang, "Quasi passive bounding of a quadruped model with articulated spine," Mechanism and Machine Theory, vol. 52, pp. 232-242, 2012.

[26] X. Wei, Y. Long, C. Wang, and S. Wang, "Rotary galloping with a lock-unlock elastic spinal joint," Proceedings of the Institution of Mechanical Engineers, Part C: Journal of Mechanical Engineering Science, vol. 229, no. 6, pp. 1088-1102, 2015.

[27] J. M. Gál, "Mammalian spinal biomechanics. II. Intervertebral lesion experiments and mechanisms of bending resistance," The Journal of Experimental Biology, vol. 174, no. 1, pp. 281-297, 1993.

[28] H. M. Herr and T. A. McMahon, "A trotting horse model," The International Journal of Robotics Research, vol. 19, no. 6, pp. 566$581,2000$.

[29] H. M. Herr and T. A. McMahon, "A galloping horse model," The International Journal of Robotics Research, vol. 20, no. 1, pp. 2637, 2001.

[30] D. P. Krasny and D. E. Orin, "Evolution of a 3D gallop in a quadrupedal model with biological characteristics," Journal of Intelligent and Robotic Systems, vol. 60, no. 1, pp. 59-82, 2010. 
[31] Z. G. Zhang, H. Kimura, and K. Takase, "Adaptive running of a quadruped robot using forced vibration and synchronization," Journal of Vibration and Control, vol. 12, no. 12, pp. 1361-1383, 2006.

[32] A. Seyfarth, M. Günther, and R. Blickhan, "Stable operation of an elastic three-segment leg," Biological Cybernetics, vol. 84, no. 5, pp. 365-382, 2001.

[33] J. E. A. Bertram and A. Gutmann, "Motions of the running horse and cheetah revisited: fundamental mechanics of the transverse and rotary gallop," Journal of the Royal Society Interface, vol. 6, no. 35, pp. 549-559, 2009.

[34] A. E. Minetti, L. P. Ardigo, E. Reinach, and F. Saibene, "The relationship between mechanical work and energy expenditure of locomotion in horses," Journal of Experimental Biology, vol. 202, no. 17, pp. 2329-2338, 1999. 


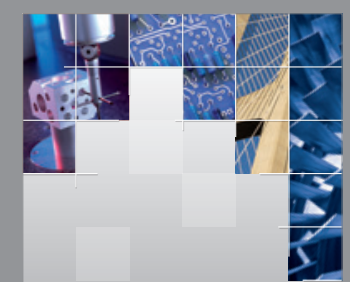

\section{Enfincering}
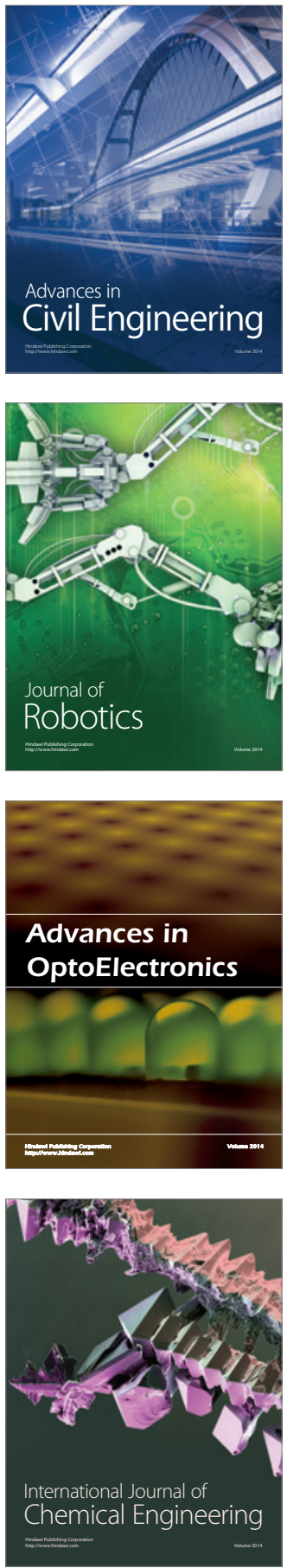

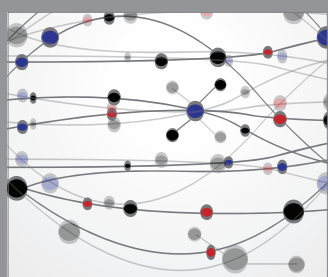

The Scientific World Journal

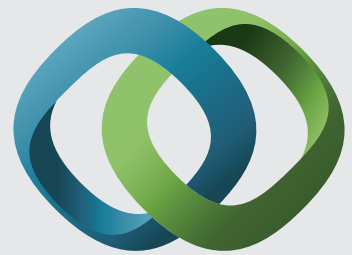

\section{Hindawi}

Submit your manuscripts at

http://www.hindawi.com
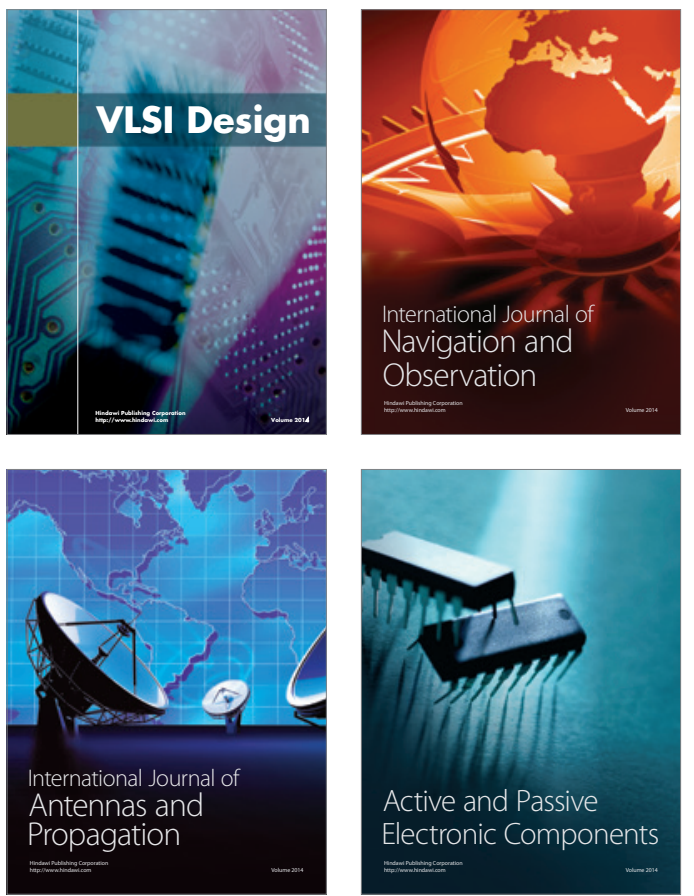
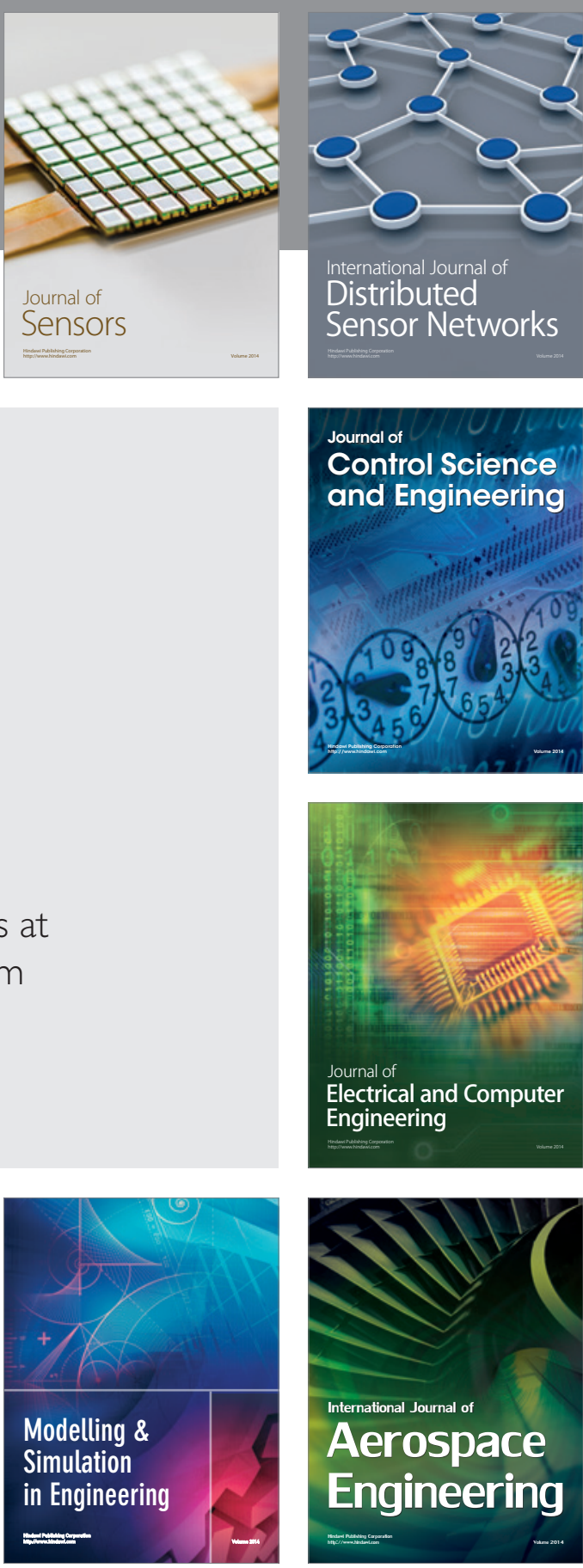

International Journal of

Distributed

Sensor Networks

Journal of

Control Science

and Engineering
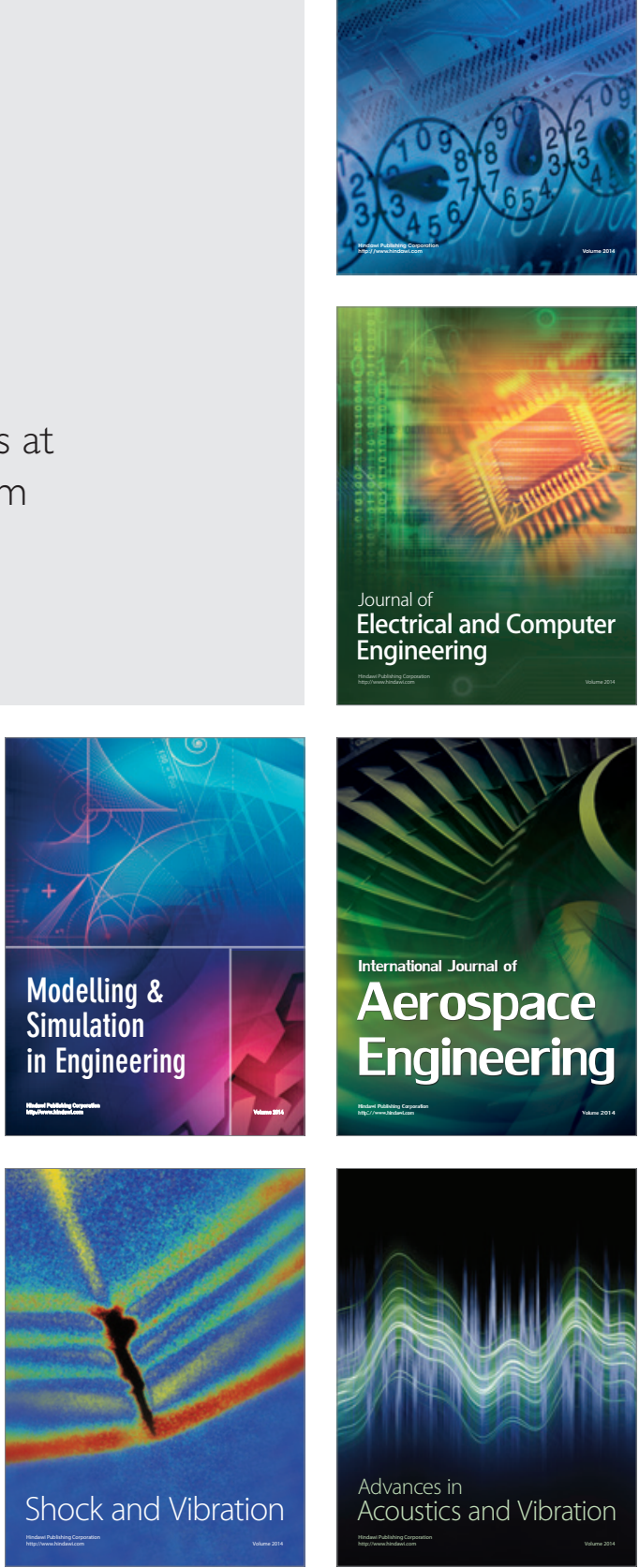